\title{
Sur quelques particularités écologiques de la faune malacologique vectrice de trématodes dans les Dallols nigériens
}

\author{
par S. GRETILLAT et G. GASTON \\ Ecole des Assistants d'Elevage de Niamey, République du Niger
}

\section{Résumé.}

Le réseau hydrographique fossile des Dallols en République du Niger est réduit pendant toute la période sèche de l'année à une série de mares alimentées par une nappe affleu:ante très légèrement salée ou par des sources d'eau douce.

La plupart de ces points d'eau ont des constantes bio-physico-chimiques propices à la vie et à la prolifération de Gastéropodes vecteurs de bilharzioses, paramphistomoses et distomatose.

Tous les ans, par suite d'une évaporation intense durant les mois de février, mars, avril et mai, non entièrement compensée par un apport d'eau suffisant, la concentration en sels dissous du milieu (chlorure, sulfate et bicarbonate de sodium), augmente pour atteindre, au bout d'un certain temps, une valeur létale, variable suivant chaque espèce de Mollusque vecteur.

En reproduisant expérimentalement des conditions analogues en laboratoire, il a été possible de déterminer les courbes de mortalité de Biomphalaria pfeifferi, Bulinus truncatus, Bulinus forskalii et Afrogyrus coretus, en fonction du taux de salinité du milieu. La DL 50 étant pour chacun d'entre eux de: 9,$6 ; 9,00 ; 1,05$ et $2,35 \mathrm{~g} /$ litre.

\section{Summary.}

Remarks on the ecological adaptation of the snail aquatic fauna in saline medium of the Dallol ponds. (République du Niger).

Human vesical and intestinal bilharziasis, bovine fasciolosis and paramphistomosis, equine gastrodiscosis and ovine carmyeriosis, are frequent in the Dallols'region, $\left(12^{\circ}-13^{\circ}\right.$ $30 \mathrm{~N}$. lat. ; $3^{\circ}$ E. long.), République du Niger, Africa. 
Dallols are fossil valleys pouring water from late Saharian lakes. They are also tributaries of the Niger River.

During the dry season, they become dry and many residual ponds of varied dimensions: from tens feet to one or two miles long, are lying along the valley bottom. The water is sometimes fresh but more frequently salt, (sodium, calcium and potassium, chlorides, sulfates, carbonates and bicarbonate), are in solution of variable proportions.

From november to april, the total salt concentration is increasing by high evaporation and the medium becomes non likely to live for aquatic vector snails, Bulinus truncatus, Biomphalaria pfeifferi, Bulinus forskalii, Lymnaea natalensis and Afrogyrus coretus.

They dead and desappear more or less early. B. pfeifferi and B. truncatus are the more resistant, (10 to $11 \mathrm{~g} / \mathrm{salt} /$ liter), L. natalensis beeing very sensible, ( 1 to $2 \mathrm{~g} / \mathrm{salt} /$ liter).

Laboratory tests in aquarium, to realise as much as possible the natural conditions, $\left(\mathrm{pH}=6\right.$ to 6,5 ; temperature: $25^{\circ}-27^{\circ} \mathrm{C}$, with good oxygenation, air-bull), with adding daily $150 \mathrm{mg} /$ liter of saline compound extract of the "sel du Fogha" (mixed saline of the soil of the Dallol Fogha), give the following results:

For each species, the salt concentration L.D. 30-35, stops the snail nutrition, when the eggs are more numerous but no able to hatch. The young snails is enlarged inside the mucilaginous wrapper. The salt concentration L.D. 50, kills all young snails.

Mortality rate, L.D. 50 and L.D. 100, for each species are indicated fig. 1; (semi logarithmical curve).

Consequently, snail population disappears yearly in the salted ponds, but lateral ponds of the valley banks supplied by fresh springs, allow the preservation of snail stocks.

The snail invasion of the dallol reappears as soon as the first rains of the raining season. Molluscs are coming out from these "estivation" lodges and are invading all the points of the river valley when the water becomes fresh.

Prophylactic programs by molluscicide products will be very easy. It is possible to obtain snail eradication in the rare permanent freshwater ponds during the dry season.

En région soudano-sahélienne, un facteur limitant la dispersion et l'importance des maladies à trématodes de l'Homme et des animaux domestiques est l'assèchement périodique des points d'eau biotopes à Mollusques vecteurs.

$\mathrm{Si}$ certaines souches de bulins peuvent résister aux dures conditions de la saison sèche en «estivant » dans la vase humide de bas-fonds de marigots (Clastrier, 1941), (Hira, 1968), cette dure période n'en amène pas moins la disparition de la presque totalité des gîtes à Gastéropodes pulmonés. De toute manière, si les survivants sont capables de réduire leurs besoins normaux en oxygène, dans la proportion de 1/11, pendant ce long séjour dans le sol (Coles, 1969), il est cependant indispensable que le milieu ne présente aucune condition létale.

Dans le cas du réseau hydrographique fossile des Dallols au Niger, reconnu comme abritant de nombreux gîtes à Gastéropodes vecteurs d'affections à trématodes (Grétillat, 1974), à ces conditions écologiques générales vient s'ajouter un facteur non moins important, l'augmentation de la concentration en sels dissous du milieu, résultat des pertes par évaporation. 
Au cours des mois très chauds de février, mars, avril et mai, le débit de la nappe affleurante alimentant les points d'eau ne suffit plus à compenser les pertes en surface, le niveau baisse et le milieu se concentre en sels dissous.

Les analyses effectuées en 1966, par Gavaud et Boulet sur des prélèvements d'eau effectués à Sabongari, au Nord de la ville de Gaya (Dallol Foga), montrent la présence dans la nappe afffeurante de sels divers: chlorures, carbonates et sulfates de sodium, potassium, calcium et magnésium. Les eaux des Dallols se dessalent, chaque année, au cours de la saison des pluies par les eaux pluviales.

Cette modification graduelle et lente des conditions physico-chimiques du biotope entraîne chaque année une destruction massive de la faune malacologique, bien avant le dessèchement complet des mares, dont les rives se recouvrent peu à peu de dépôts alluvionnaires salins renfermant ces divers sels.

Ayant remarqué que certaines espèces résistaient beaucoup plus longtemps que d'autres à ce changement de milieu, il nous a paru intéressant de reconstituer expérimentalement, en aquarium, au laboratoire, les conditions rencontrées sur le terrain et de comparer leurs possibilités d'adaptation ou de résistance respectives, à une élévation progressive de la concentration en sels dissous de l'eau du gîte.

Sur les cinq espèces de Gastéropodes rencontrées, au cours de prospections, dans les Dallols nigériens, quatre seulement ont été mises en élevage pour être soumises à une série de tests.

Ce sont: Biomphalaria pfeifferi (Krs.), Bulinus truncatus (Clessin), Bulinus forskalii (Ehrenb.), et Afrogyrus coretus (Blainville). Lymnaea natalensis Krs., n'a fait l'objet d'aucun test au cours de cette expérimentation, car difficile à maintenir en élevage à Niamey, il s'est, d'autre part, révélé extrêmement sensible aux sels de sodium (dose léta!e: 1 à $2 \mathrm{~g}$ /litre), chiffre que signale d'ailleurs Ezzat, en 1961, pour ce Mollusque, dans le Delta du Nil en Egypte.

\section{Protocole expérimental}

Pour recréer au laboratoire dans toute la mesure du possible les conditions rencontrées sur le terrain, les Mollusques sont placés dans des aquariums auxquels sont ajoutés quotidiennement $150 \mathrm{mg}$ /litre d'un mélange de sels de sodium (chlorure, sulfate et bicarbonate), en provenance du Dallol Foga (village de Kota Koté, à $25 \mathrm{~km} \mathrm{au}$ Nord de Gaya). On obtient ainsi une augmentation progressive de la concentration en sels dissous du milieu à peu près semblable à celle qui a lieu au cours de l'évaporation des mares pendant la saison chaude.

La concentration de 7 à $8 \mathrm{~g} /$ litre est atteinte au bout de 8 à 9 semaines (deux mois), qui correspond, à peu près, à la période de baisse des eaux en mars-avril en année normale, ou en février-mars en année moins pluvieuse.

Pour faciliter les manipulations, c'est une solution mère à $3 \%$ qui est ajoutée quotidiennement à l'eau des aquariums de deux litres, de pH 6 à 6,5, fortement aérée 
et dont la température varie entre 25 et $27^{\circ} \mathrm{C}$. Elle est préparée à partir de "sel » acheté sur place au village de Kota Koté, extrait des alluvions sableuses du Dallol Foga par les sauniers locaux.

La terre salée des rives est placée dans des trémies en osier pour y être lessivée par des eaux de lavage qui, après les avoir épuisées, sont portées à l'ébullition pour récupérer un dépôt cristallisé, de couleur marron foncé, appelé «Sel de Foga », consommé sur place ou exporté vers le Nord par caravanes.

Après avoir enlevé le sable et l'argile par ébullition et décantation d'une solution concentrée de ce mélange, on la traite à l'éther de pétrole pour la débarrasser des matières organiques qu'elle contient encore. On procède alors à une dessication sous vide pour obtenir un mélange cristallisé renfermant approximativement, si l'on se rapporte aux dosages effectués par Gavaud et Boulet en 1968, sur les dépôts salins des rives du Dallol Foga à Sabongari, à quelques kilomètres de Kota Koté :

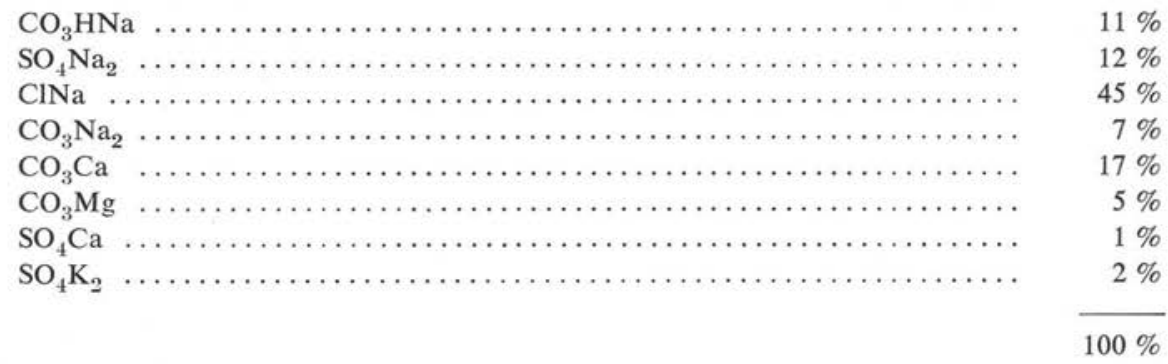

Les essais ont été faits sur 50 spécimens adultes de Biomphalaria pfeifferi, 50 Bulinus truncatus, 45 Afrogyrus coretus et 33 Bulinus forskalii, souches en provenance des Dallols Foga et Bosso. Pour chaque espèce, des aquariums témoins contenant chacun 25 spécimens adultes sont maintenus en eau douce pour contrôle de mortalité dans les conditions d'élevage en laboratoire.

\section{Résultats}

Pour les quatre espèces mises en expérience, les résultats sont représentés par quatre courbes de mortalité mettant nettement en évidence leurs résistances particulière et spécifique.

Biomphalaria pfeifferi et Bulinus truncatus sont beaucoup moins sensibles à une augmentation de la salinité du milieu que Bulinus forskalii et Afrogyrus coretus.

Pour tous les quatre, cependant, existe un seuil de morbidité correspondant à un taux de mortalité de $30 \%$ au-delà duquel les Mollusques ne s'alimentent plus.

Avant que ce seuil soit atteint, on assiste pendant quelques jours à une accélération du rythme des pontes. 
A ce sujet, il est intéressant de noter que, si les jeunes éclosent et se développent à peu près normalement dans un milieu d'un taux létal de 30 à $35 \%$, il leur est difficile, voire impossible, de se libérer de la gangue mucilagineuse, substratum de la ponte, qui les entoure, quand la concentration en sels dissous est plus élevée. Il doit s'agir d'un processus où intervient la pression osmotique, car ces mêmes pontes éclosent spontanément quand on les place en eau douce. Les produits ainsi obtenus ont une taille très supérieure à celle de ceux obtenus normalement dans les aquariums témoins. Cela semble prouver que, s'il y a retard à l'éclosion, la croissance du jeune a lieu quand même à l'intérieur de son alvéole.

A un taux de salinité correspondant à la DL 50 de l'espèce apparaît une diminution des possibilités de multiplication, suivie d'un arrêt brusque des pontes, ou parfois les dernières pouvant être stériles ou monstrueuses. Le développement des jeunes est stoppé et suivi de mort.

Cette DL 50 est de $9,6 \mathrm{~g} / 1$ pour $B$. pfeifferi, $9,00 \mathrm{~g} / 1$ pour B. truncatus, 1,05 g/1 pour $B$. forskalii et $2,35 \mathrm{~g} / 1$ pour $A$. coretus. Pour cette dernière espèce, des taux très faibles $(1,20 \mathrm{~g} / 1)$ sont létaux pour les pontes, les jeunes étant tués en 24 heures dans leurs alvéoles (fig. 1).

\section{Interprétation des résultats}

En valeur absolue, les chiffres trouvés sont très contestables, car :

$1^{\circ}$ la composition exacte du mélange salin utilisé n'est pas connue et très complexe ;

$2^{\circ}$ les conditions dans lesquelles sont placés les Mollusques sont artificielles et ne sont pas celles rencontrées dans les eaux des Dallols.

Ils peuvent cependant être utilisés par comparaison entre les différentes espèces et surtout avec ceux trouvés sur le terrain.

Biomphalaria pfeifferi et Bulinus truncatus sont les derniers Gastéropodes à disparaitre, vers le mois de mars, dans les Dallols et on les trouve encore vivants et actifs dans des eaux renfermant 6 à $7 \mathrm{~g} / 1$ de sels dissous dans des gîtes à Sambon Birni, à $12 \mathrm{~km}$ au Nord de Gaya (Dallol Foga). Par contre, dans des mares titrant 10 à $11 \mathrm{~g} / \mathrm{l}$, à Sambongari, on ne trouve plus que des coquilles vides de planorbes.

$\mathrm{Au}$ point de vue épidémiologique, ces remarques écologiques sont intéressantes. Elles démontrent une destruction annuelle de la faune malacologique des Dallols qui ne peut être reconstituée à chaque saison des pluies qu'à partir des gîtes permanents en eau douce situés en général sur les flancs de la vallée et inondés lors de la crue d'hivernage.

Ces considérations d'ordre épidémiologique pourraient être très utiles dans l'élaboration de campagnes prophylactiques de lutte contre les affections à trématodes de ces régions. 


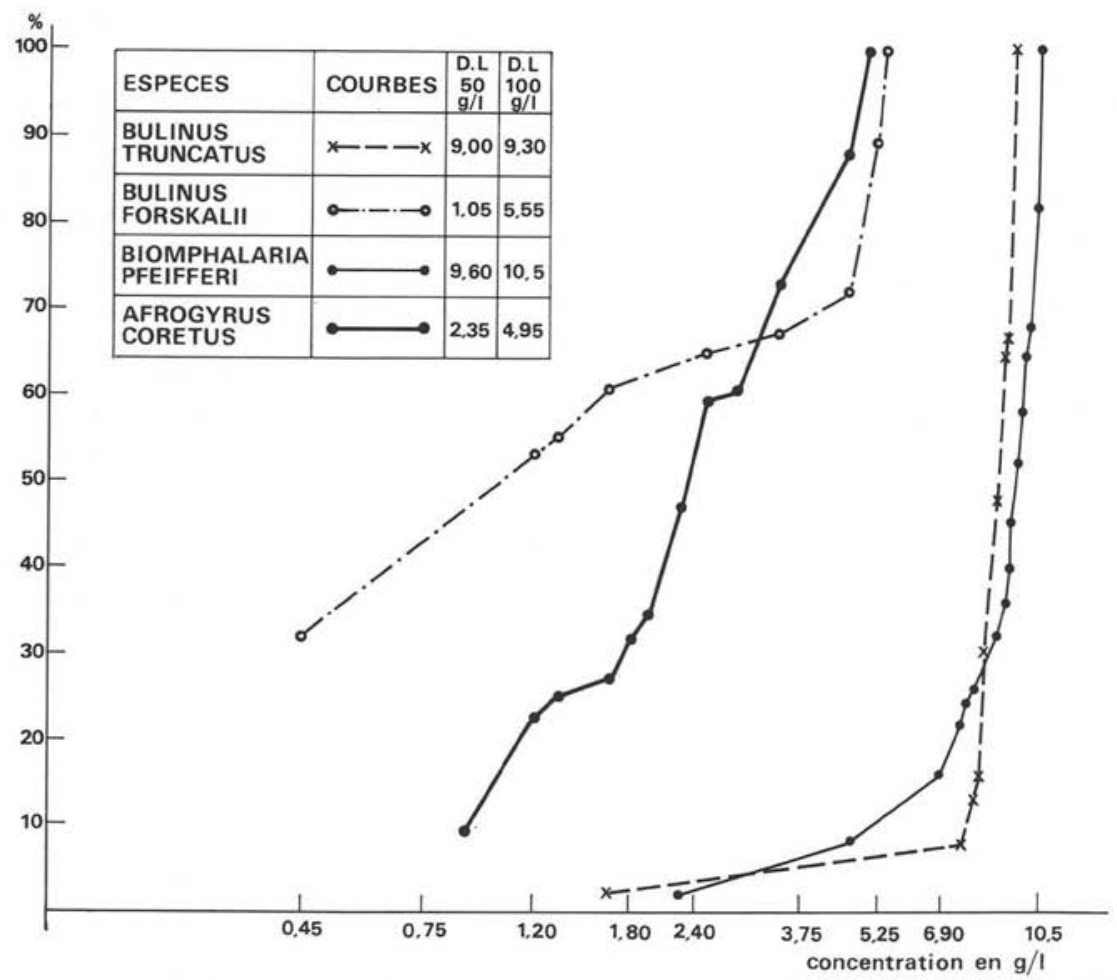

Fig. 1. - Courbes de mortalité de Biomphalaria pfeifferi, Bulinus truncatus, Bulinus forskalii et Afrogyrus coretus en fonction de la concentration en sels dissous du milieu (Coordonnées semi-logarithmiques). Taux de mortalité des lots témoins en fin d'expérience : Biomphalaria pfeifferi : $8 \%$ au bout de 67 jours; Bulinus truncatus : $6 \%$ au bout de 60 jours; Bulinus forskalii: $15 \%$ au bout de 29 jours; Afrogyrus coretus : $8 \%$ au bout de 27 jours.

\section{REMERCIEMENTS}

- Ce travail réalisé dans les laboratoires de biologie et de physique de l'Ecole a pu être mené à bien grâce à la collaboration technique des élèves de $2^{\circ}$ année, MM. Doulla Biga et Halidou Amadou.

- Les souches de Mollusques ayant servi à cette expérimentation ont été déterminées par M. le Pr Mandhal-Barth, du Danish Bilharziasis Laboratory de Charlottenlund Denmark, que nous remercions bien vivement.

\section{Bibliographie}

Clastrier (J.), 1941. - Prophylaxie de la bilharziose. Sur la résistance des bulins à la dessication. Arch. I.P. Algérie, 19, 64-66. 
Coles (G. C.), 1969. - Observations on weight loss and oxygene uptake of estivating Bulinus nasutus an intermediate host of Schistosoma haematobium. Ann. trop. Med. Parasit., 63, 393-398.

Ezzat (M. A. E.), 1961. - The salinity of water and its effect on the vitality of Lymnaea caillaudi the Snail intermediate host of Fasciola gigantica in Egypt. J. of the Arab. Vet. Med. Assoc., 21, 177-183.

Gavaud (M.) et Boulet (R.), 1966. - Etude pédologique du Niger central (1 c. 1/500.000). O.R.S.T.O.M., Dakar.

HIR I (P. R.), 1968. - Study on the capability of the Snail transmiting the urinary schistosomiasis in Western Nigeria to survive dry conditions. W. Afr. Med. J., 17, 153-160. 